\title{
WEAK PARTITION PROPERTIES FOR INFINITE CARDINALS. I
}

\author{
E. M. KLEINBERG
}

Abstract. Partition properties are perhaps the most fruitful of the various methods for defining and discussing large cardinals in set theory. In this paper we weaken in a natural way the most well known of these partition properties and examine the extent to which the cardinals defined remain "large."

1.

1.1. An area of set theory which has come under a great deal of study recently is that concerned with partition properties for cardinal numbers. In order to consider several specific examples let $\kappa$ denote an uncountable cardinal and let us introduce $\kappa \rightarrow(\kappa)^{2}\left(\kappa \rightarrow(\kappa)^{<\omega}\right)$ to denote $\left(^{*}\right)$ for each partition ${ }^{1}$ of the 2 element (finite) subsets of $\kappa$ into two pieces there exists a subset $x$ of $\kappa$ of cardinality $\kappa$ such that the 2 element (such that for each $i$, the i-element) subsets of $x$ are contained in only one of the pieces. Clearly $\kappa \rightarrow(\kappa)^{<\omega}$ implies $\kappa \rightarrow(\kappa)^{2}$. The following are several well-known results concerning these relations:

R1 If $\kappa$ is a measurable cardinal then $\kappa \rightarrow(\kappa)^{<\omega}$.

R2 If there exists a $\kappa$ such that $\kappa \rightarrow(\kappa)^{<\omega}$, then there exists a nonconstructible $\Delta_{3}^{1}$ set of integers.

R3 If $\kappa \rightarrow(\kappa)^{2}$, then $\kappa$ is a strongly inaccessible cardinal.

R4 For $\lambda$ an infinite cardinal less than $\kappa$, let $\kappa \rightarrow(\kappa)_{\lambda}^{2}\left(\kappa \rightarrow(\kappa)_{\lambda}^{<\omega}\right)$ denote the assertion $\left({ }^{*}\right)$ changed to cover partitions into $\lambda$ pieces.

Then for each infinite cardinal $\lambda$ less than $\kappa, \kappa \rightarrow(\kappa)_{\lambda}^{2}\left(\kappa \rightarrow(\kappa)_{\lambda}^{<\omega}\right)$ is equivalent to $\kappa \rightarrow(\kappa)^{2}\left(\kappa \rightarrow(\kappa)^{<\omega}\right)$.

1.2. Now R4 suggests a natural way to try to weaken $\kappa \rightarrow(\kappa)^{2}$ and $\kappa \rightarrow(\kappa)^{<\omega}$. For instance, given a partition of the 2 element subsets of $\kappa$ in $\lambda$ pieces, $\kappa \rightarrow(\kappa)_{\lambda}^{2}$ states that there exists a set $x$ of cardinality $\kappa$ all of whose 2 element subsets are contained in only one of the pieceswhat if we just assert that there exists a subset $x$ of $\kappa$ of cardinality $\kappa$ all of whose 2 element subsets are contained in fewer than $\lambda$ of the pieces? We make this notion precise by introducing $\kappa \stackrel{w}{\rightarrow}(\kappa)^{2}\left(\kappa \stackrel{w}{\rightarrow}(\kappa)^{<\omega}\right)$ to denote for each infinite cardinal $\lambda$ less than $\kappa$ and partition of the 2

Received by the editors March 13, 1968 and, in revised form, January 24, 1970.

AMS 1970 subject classifications. Primary 02K35.

Key words and phrases. Partition property, cofinality, strongly inaccessible cardinal, Ramsey cardinal.

${ }^{1}$ If $P$ and $Q$ are sets, we will think of a partition of $P$ into $Q$ pieces as a function from $P$ into $Q$. 
element (finite) subsets of $\kappa$ into $\lambda$ pieces, there exists a subset $x$ of $\kappa$ of cardinality $\kappa$ and a cardinal $\delta$ less than $\lambda$ such that the 2 element (such that for each $i$, the $i$-element) subsets of $x$ are contained in at most $\delta$ of the pieces.

Our two main results, then, are as follows:

THEOREM 1. If $\kappa \stackrel{w}{\rightarrow}(\kappa)^{2}$ then $\kappa$ is a strongly inaccessible cardinal.

THEOREM 2. $\kappa \stackrel{w}{\rightarrow}(\kappa)^{<\omega}$ and $\kappa \rightarrow(\kappa)^{<\omega}$ are equivalent.

Thus of the partition properties for $\kappa$ given by the weakened assertions, the first is still strong enough to render $\kappa$ inaccessible, and the second is, in fact, just as strong as its original counterpart.

Along the way to proving Theorems 1 and 2 we shall establish several lemmas and related results which are of interest in their own right. Among these we include a characterization, in terms of partition relations, of the concept of cofinality (for cardinals).

2. Throughout this section $\kappa$ will denote an uncountable cardinal, and $\lambda \geqq \delta$ will denote cardinals less than $\kappa$. In order to facilitate our presentation we introduce, for any $i<\omega, \kappa \rightarrow(\kappa)_{\lambda, \delta}^{i}\left(\kappa \rightarrow(\kappa)_{\lambda, \delta}^{<\omega}\right)$ to denote for each partition of the i-element (finite) subsets of $\kappa$ into $\lambda$ pieces there exists a subset $x$ of $\kappa$ of cardinality $\kappa$ such that the $i$-element (such that for each $i$, the $i$-element) subsets of $x$ are contained in at most $\delta$ of the pieces. ${ }^{2}$

The cofinality of $\kappa, \operatorname{cf}(\kappa)$, is the least cardinal which can be mapped onto an unbounded subset of $\kappa$. The following lemma is of interest in its own right:

LEMMA 1. $\kappa \rightarrow(\kappa)_{\lambda, \delta}^{1}$ iff $\lambda<\operatorname{cf}(\kappa)$ or $\delta \geqq \operatorname{cf}(\kappa)$.

Proof. $(\Rightarrow)$. Suppose that $\delta<\operatorname{cf}(\kappa) \leqq \lambda$, and let $g$ map $\lambda$ onto an unbounded subset of $\kappa$. With $f: \kappa \rightarrow \lambda$ given by $f(\eta)={ }_{\mathrm{d} f}$ "the least $\xi<\lambda$ such that $g(\xi)>\eta, " \kappa \rightarrow(\kappa)_{\lambda, \delta}^{1}$ presents us with a subset $x$ of $\kappa$ of cardinality $\kappa$ such that the range of $f$ on $x$ has cardinality at most $\delta$. (We use the notation, henceforth, that $|y|$ is the cardinality of the set $y$ and $h^{\prime \prime} y$ is the range of the function $h$ on the set $y$.) Since now $\left|(g \circ f)^{\prime \prime} x\right| \leqq \delta<\operatorname{cf}(\kappa), \quad U(g \circ f)^{\prime \prime} x<\kappa$, and so for some $\eta$ in $x$, $U(g \circ f)^{\prime \prime} x<\eta$. But now $g(f(\eta)) \leqq \eta$ in contradiction to the definition of $f$.

$(\Leftarrow)$. Suppose that $\kappa=U_{\xi<\lambda} A_{\xi}$. Then if $\lambda<\operatorname{cf}(\kappa)$, some $A_{\xi}$ would have power $\kappa$ and hence be, in fact, 1-homogeneous for the partition $\left(\left\{A_{\xi} \mid \xi<\lambda\right\}\right)$. On the other hand, suppose that $\operatorname{cf}(\kappa) \leqq \delta$, and let $g$

The set $x$ mentioned in this definition is said to be $\delta$-homogeneous for the partition. 
map $\delta$ onto an unbounded subset of $\kappa$. If the cardinalities of the $A_{\xi}$ were bounded in $\kappa$ by, say, $\gamma$, we would have the cardinality of $\bigcup_{\xi<\lambda} A_{\xi}$ at most that of $\gamma \cdot \lambda$, which is less than $\kappa$, a contradiction. So for each $\eta<\delta$, let $A_{h(\eta)}$ have cardinality at least that of $g(\eta)$. Then $\mathrm{U}_{\eta<\delta} A_{h(\eta)}$ is an appropriate $\delta$-homogeneous set for the partition.

An uncountable cardinal $\gamma$ is said to be strongly inaccessible if (1) $\operatorname{cf}(\gamma)=\gamma$ and (2) $\xi<\gamma$ implies $|2 \xi|<\gamma$. We shall establish Theorem 1 as follows:

Suppose that $\kappa \stackrel{w}{\rightarrow}(\kappa)^{2}$. If $\operatorname{cf}(\kappa)<\kappa$, let $f: \kappa \rightarrow \operatorname{cf}(\kappa)$ be as given in the proof of Lemma 1. Then by $\kappa \stackrel{w}{\rightarrow}(\kappa)^{2}$ there exist $\delta<\operatorname{cf}(\kappa)$ and $x \subseteq \kappa$ such that $|x|=\kappa$ and $\left|f^{\prime \prime} x\right| \leqq \delta$. As shown in the proof of Lemma 1 , this yields a contradiction. We are thus forced to conclude that $\operatorname{cf}(\kappa)=\kappa$. In a similar way, the proof of Lemma 2 will show that there is no least $\lambda<\kappa$ such that $\left|2^{\lambda}\right| \geqq \kappa$.

LEMmA 2. If $\kappa \rightarrow(\kappa)_{\lambda, \delta}^{2}$ and $\left|2^{\lambda}\right| \geqq \kappa$, then $\left|2^{\delta}\right| \geqq \kappa$.

Proof. Suppose that $\kappa \rightarrow(\kappa)_{\lambda, \delta}^{2}$ and $\left|2^{\lambda}\right| \geqq \kappa$. Let $F$ map $\kappa$ 1-1 into $2^{\lambda}$ and let our partition of the two element subsets of $\kappa$ into $\lambda$ pieces (viewed as a function from the two element subsets of $\kappa$ into $\lambda$ ) send any pair $\{\alpha, \beta\}$ to the least place at which $F(\alpha)$ and $F(\beta)$ differ (in the sense of functions from $\lambda$ into 2). Then if $x$ is the set given by $\kappa \rightarrow(\kappa)_{\lambda, \delta}^{2}$, and if $A$ denotes the range of the partition on the two element subsets of $x$, the map $R: x \rightarrow 2^{\lambda}$ given by $R(\alpha)=F(\alpha)\lceil A$ is $1-1$ and has a range of power $\left|2^{A}\right|$. Since $|A| \leqq \delta$ and $|x|=\kappa$, this gives $\left|2^{\delta}\right| \geqq \kappa$.

REMARK. The above lemma remains true if $\left|2^{\lambda}\right|$ and $\left|2^{\delta}\right|$ are replaced by $\lambda^{+}$and $\delta^{+}$, respectively. (Recall that for ordinals $\gamma, \gamma^{+}$ denotes the successor cardinal to $|\gamma|$.) To see this, suppose that $F$ maps $\kappa$ 1-1 into $\lambda^{+}$, and, that for each $\eta<\lambda^{+}, f_{\eta}$ maps $\eta 1-1$ into $\lambda$. In partitioning this time, send $\{\alpha, \beta\}$ to $f_{F(\alpha)}(F(\beta))$ or $f_{F(\beta)}(F(\alpha))$, whichever is defined, and let $x$ and $A$ be as before. Then if $\alpha<\beta<\eta$ are members of the range of $F$ on $x, f_{\eta}(\alpha)$ and $f_{\eta}(\beta)$ are distinct members of $A$. Thus any initial segment of $F^{\prime \prime} x$ has cardinality at most that of $A$, and so $\kappa=|x|=\left|F^{\prime \prime} x\right| \leqq|A|+\delta^{+}$.

Proof of Theorem. 2. In light of R4 it is clear that $k \rightarrow(\kappa)^{<\omega}$ implies $\kappa \stackrel{w}{\rightarrow}(\kappa)^{<\omega}$. We now demonstrate the converse of this: Suppose that $F$ is a given partition of the finite subsets of $\kappa$ into two pieces. We define a new partition, $G$, of the finite subsets of $\kappa$ into $\omega$ pieces as follows: let, for each positive integer $i, p_{i}$ denote the $i$ th largest prime number greater than or equal to 2 . On the finite subsets of $k$ whose number of elements is not a power of a prime we define $G$ to be identically 0 . Suppose that $q$ is a subset of $\kappa$ containing $p_{i}^{n}$ elements. 
Then if $m$ is the largest integer $k$ such that $k \cdot i \leqq p_{i}^{n}$ we can list the members of $q$, in increasing order, as $q_{1}, \cdots, q_{i}, q_{i+1}, \cdots, q_{2 i}, \cdots$, $q_{(m-1) i}, \cdots, q_{m i}, \cdots, q_{p_{i}}^{n}$. Let

$$
G(q)={ }_{\mathrm{df}} 2^{F\left(\left\{q_{1}, \ldots, q_{i}\right\}\right)} 3^{F\left(\left\{q_{i+1}, \ldots, q_{2 i}\right\}\right) \ldots p_{m} F\left(\left\{q_{(m-1) i}, \ldots, q_{m i}\right\}\right) .}
$$

Now as given by $\kappa \stackrel{w}{\rightarrow}(\kappa)^{<\omega}$, let $x$ be a subset of $\kappa$ of cardinality $\kappa$ and let $n_{0}$ be a positive integer such that for each $i$, the range of $G$ on the $i$-element subsets of $x$ has cardinality at most $n_{0}$.

Claim. For each positive integer $i$ there exists an ordinal $\alpha$ less than $\kappa$ such that the range of $F$ on the $i$-element subsets of $x-\alpha$ has cardinality 1.

(Proof. Suppose that this claim is not true for the positive integer $i$. Let $n$ and $k$ be integers satisfying $k \cdot i \leqq p_{i}^{n}$ and $2^{k}>n_{0}$. Then it is routine to see that the range of $G$ on the $p_{i}^{n}$-element subsets of $x$ has cardinality at least $2^{k}$. Since $2^{k}>n_{0}$, this is in contradiction to our initial choice of $x$ and $n_{0}$. The claim is thus established.

Now for each positive integer $i$ let $\alpha_{i}$ be the least ordinal satisfying the conclusion of the claim. Then since (as a result of Theorem 1) the cofinality of $\kappa$ is $\kappa, \bigcup_{i<\omega} \alpha_{i}<\kappa$. We thus have that $x-U_{i<\omega} \alpha_{i}$ is a subset $z$ of $\kappa$ of cardinality $\kappa$ such that for each $i<\omega$ the range of $F$ on the $i$-element subsets of $z$ has cardinality 1 . The original partition $F$ was arbitrary and so the theorem is proved.

\section{REFERENCES}

1. P. Erdös and A. Hajnal, On the structure of set-mappings, Acta Math. Acad. Sci. Hungar. 9 (1958), 111-131. MR 20 \#1630.

2. - Some remarks concerning our paper "On the structure of set mappings," Acta Math. Acad. Sci. Hungar. 9 (1958), 223-226.

3. E. M. Kleinberg, Somewhat homogeneous sets. I, Notices Amer. Math. Soc. 16 (1969), 840. Abstract \#69T-E49.

4. - Somewhat homogeneous sets. II, Notices Amer. Math. Soc. 16 (1969), 1088. Abstract \#69T-E89.

5. - Somewhat homogeneous sets. III, Notices Amer. Math. Soc. 17 (1970), 296. Abstract \#70T-E9.

6. - Strong partition properties for infinite cardinals, Doctoral Dissertation, Rockefeller University, New York, 1969.

7. J. Silver, Some applications of model theory in set theory, Doctoral Dissertation, University of California, Berkeley, Calif., 1966.

8. R. M. Solovay, A nonconstructible $\Delta_{3}^{1}$ set of integers, Trans. Amer. Math. Soc. 127 (1967), 50-75. MR $35 \# 2748$.

Rockefeller University, New York, New York 10021

Massachusetts Institute of Technology, Cambridge, Massachusetts 02139 